\title{
Jieduan-Niwan Formula Reduces Liver Apoptosis in a Rat Model of Acute-on-Chronic Liver Failure by Regulating the E2F1-Mediated Intrinsic Apoptosis Pathway
}

\author{
Wenlong Yang, Yulin Hao, Weixin Hou, Xian Fang, Peng Fang, Tianyuan Jiang, \\ Chongyang Ma, and Qiuyun Zhang \\ Beijing Key Lab of TCM Collateral Disease Theory Research, School of Traditional Chinese Medicine, Capital Medical University, \\ Beijing, China \\ Correspondence should be addressed to Qiuyun Zhang; zhangqiuyun8202@aliyun.com
}

Received 15 May 2019; Revised 28 July 2019; Accepted 24 August 2019; Published 11 November 2019

Academic Editor: Marisa Colone

Copyright $\odot 2019$ Wenlong Yang et al. This is an open access article distributed under the Creative Commons Attribution License, which permits unrestricted use, distribution, and reproduction in any medium, provided the original work is properly cited.

\begin{abstract}
Acute-on-chronic liver failure (ACLF) is a serious and complicated disease that threatens human health because its pathogenesis is unclear, and the outcome of the current therapies has been less than satisfactory. A national famous doctor of traditional Chinese medicine, Qian Ying, created the Jieduan-Niwan Formula (JDNW), based on his long-term clinical experience. However, despite the good clinical outcome, the biological mechanism by which it works is unknown. In the current study, we established an ACLF rat model by administering human serum albumin (HSA) combined with D-galactosamine (D-GalN) and lipopolysaccharide (LPS) to explore the potential mechanism of JDNW in treating ACLF. The rats were treated with JDNW by administration of the model substances and sacrificed after 4,8 , and $12 \mathrm{~h}$. Then we divided the rats into normal group, model at $4 \mathrm{~h}$, model at $8 \mathrm{~h}$, model at $12 \mathrm{~h}, \mathrm{JDNW}$ at $4 \mathrm{~h}, \mathrm{JDNW}$ at $8 \mathrm{~h}$, and JDNW at $12 \mathrm{~h}$. Biochemical and histopathological examinations were performed to compare the rats in different groups. Compared with the ACLF model group, expression levels of alanine transaminase, aspartate aminotransferase, total bilirubin, and TNF- $\alpha$ and IL-6 proteins were reduced in the JDNW group at the corresponding time points, the survival rates of rats were increased, and the pathological condition of the liver was improved. In addition, JDNW treatment improved the ultrastructure of hepatocytes and mitochondria and decreased the hepatocyte apoptosis index. E2F1, P53, P73, Apaf-1, p14ARF, caspase-3, caspase-6, and caspase-7 levels in the JDNW group were distinctly lower than those in the untreated rats. Moreover, Bcl-2 and Mcl-1 levels increased. Thus, JDNW decreases ACLF-induced mortality in rats by modulating the E2F1-mediated intrinsic apoptotic pathway.
\end{abstract}

\section{Introduction}

Acute-on-chronic liver failure (ACLF) is a newly discovered clinical syndrome, characterized by early chronic liver disease or cirrhosis with organ failure. The 28-day mortality rate is high (50-90\%) [1]. Predisposing factors to develop the syndrome may be intrahepatic or extrahepatic, and potential chronic liver injury may occur whether or not the patient develops cirrhosis [2]. Once cirrhosis has transitioned from the compensated to the decompensated period, the short-term survival is 3-5 years. At this point, it is recommended to evaluate liver transplantation, except in the presence of contraindications [3]. Despite advances in medical treatment, clinical management of ACLF remains limited and challenging. When medical treatment fails, liver transplantation is the only option to save the patient's life [4].

However, traditional Chinese medicine has shown its superiority over conventional ACLF treatments owing to its multitarget, integrity effects with few side effects. A multicenter and randomized controlled trial demonstrated that a combination of Chinese and Western Medicine is effective for the treatment of ACLF, with a low mortality rate and better efficacy [5]. The Jieduan-Niwan formula (JDNW), which originated from the national celebrated traditional Chinese medicine expert Qian Ying, had a significant impact 
on this disease. In a clinical study, about 110 selected patients were administered the combined treatment of JDNW and Western Medicine or the Western Medicine alone. The combined treatment showed superior results in reducing mortality and in improving liver function and other symptoms as compared to the Western Medicine control group [6].

Apoptosis is a mechanism of programmed cell death and is essential for organism development and tissue homeostasis. The occurrence and onset of liver failure are closely related to apoptosis and inflammation [7]. Our previous studies showed that JDNW formula could prolong liver survival time and ameliorate its injury, which might be related to reduced levels of inflammatory cytokines, apoptotic index, and liver cell proliferation [8-14]. JDNW could stimulate the compensatory proliferation of hepatocytes by regulating the expression of E2F1 [15]. However, the main aim of this study was to investigate whether JDNW could reduce liver apoptosis in a rat model of ACLF, and if this mechanism was mediated by the E2F1 intrinsic pathway.

\section{Materials and Methods}

2.1. Reagents. Human serum albumin (HSA, A9731-5G), D-Galactosamine (D-GalN, G0500-25G), and Lipopolysaccharide (LPS, 109K4075) were purchased from SigmaAldrich (St. Louis, MO, USA). Colorimetric TUNEL Apoptosis Assay Kit was purchased from Beyotime (C1098). ELISA kits for Rat IL-6 (CRE005) and Rat TNF- $\alpha$ (CRE003) were purchased from 4A Biotech Co., Ltd.

\subsection{Preparation and High-Performance Liquid Chromatog-} raphy (HPLC) Analysis of JDNW. DNW was prepared from ten commonly used herbs (Table 1): Phyllanthus urinaria Linn., Radix Astragali, Fructus Trichosanthis, Herba Lysimachiae, Herba Visci, Radix et Rhizoma Notoginseng, Rhizoma Curcumae, Radix et Rhizoma Salviae Miltiorrhizae, Radix Rehmanniae, and Radix Aconiti Lateralis Preparata. The raw herbs were purchased from the Beijing Tong Ren Tang Group Co. Ltd. (Beijing, China). They were mixed in the ratio of $30: 30: 30: 30: 30: 6: 6: 20: 20: 15$ (dry weight in grams). All herbs were decocted twice. The two cycles of filtered suspensions were mixed and concentrated to $50 \mathrm{~mL}$ (with a density of $4.34 \mathrm{~g} / \mathrm{mL}$ ) with a vacuum rotary evaporator. Subsequently, the aqueous solution was stored at $4^{\circ} \mathrm{C}$ and heated to $37^{\circ} \mathrm{C}$ in a water bath before use. For quality control purposes, salvianolic acid $\mathrm{B}$ (the representative component of JDNW) was determined by HPLC. The results are shown in Figure 1.

2.3. Animal Model and Treatment. Male Wistar rats, weighing 180-200 g, were obtained from the Academy of Military Medical Sciences Laboratory Animal Center (Beijing, China). License number was SCXK-(Beijing) 20160006. Animals were housed in a specific pathogen-free environment. All protocols were approved by the Animal Experiments and Experimental Animal Welfare Committee of Capital Medical University (Beijing, China).
The ACLF rat model we used was established as described previously [16]. Briefly, ACLF was induced by combined administration of human serum albumin (HSA) with D-galactosamine (D-GalN) and lipopolysaccharide (LPS). Rats were divided into two model stages (Figure 2). (1) Liver cirrhosis model stage: the rats $(n=150)$ were randomized into two groups, the control group $(n=10)$ and the treatment group $(n=140)$. The former group was given multiple subcutaneous injections of $0.5 \mathrm{~mL}$ saline solution; meanwhile the latter one was injected with one dose of HSA (containing $4 \mathrm{mg}$ ) for sensitization, followed by administration of $0.5 \mathrm{~mL}$ HSA into the tail vein twice a week. Six weeks later, the successful establishment of models was confirmed by Masson's trichrome staining (see Supplementary Material here). Finally, 98 liver cirrhosis model rats were randomly divided into ACLF model and JDNW groups with 49 rats in each group. (2) ACLF model stage: model and JDNW groups were given acute attack induced by simultaneous administration of $400 \mathrm{mg} / \mathrm{kg} \mathrm{D-GalN}$ and $100 \mu \mathrm{g} / \mathrm{kg}$ LPS into the enterocoelia. Rats in the control group received the same volume of saline solution. On the other hand, rats of the JDNW group were administered $21.7 \mathrm{~g} / \mathrm{kg} / \mathrm{d}$ JDNW decoction via oral gavage twice a day, for 3 days, which was the optimized dosage-regimen according to our preliminary study [17].

Each group of rats was randomly divided into 3 subgroups and sacrificed at 4, 8 , and $12 \mathrm{~h}$, respectively, following D-GalN and LPS administration. After intraperitoneal injection of $3 \%$ sodium pentobarbital anesthetic $(30 \mathrm{mg} / \mathrm{kg})$, blood was collected from the aorta and used for biochemical analyses. Furthermore, the liver was removed, and the right lobe was fixed in $10 \%$ neutral formalin buffer solution and embedded in paraffin for pathological staining. Additionally, a small portion of liver tissue from the ipsilateral side was fixed with $2.5 \%$ glutaraldehyde solution for electron microscopy, and other lobes were dissected and frozen in liquid nitrogen for further proteomic study.

2.4. Blood Sample Examination. Blood was centrifuged for $15 \mathrm{~min}$ at $4^{\circ} \mathrm{C}$ and $3000 \mathrm{rpm}$ and serum was collected. The serum levels of alanine transaminase (ALT), aspartate transaminase (AST), and total bilirubin (TBIL) were detected by using a Hitachi 7600 Automatic Analyzer (Hitachi, Inc., Japan).

2.5. ELISA of the Hepatic Tissue. The liver tissue was homogenized using $10 \mathrm{~mL}$ of PBS for $1 \mathrm{~g}$ of tissue and centrifuged at $4500 \mathrm{rpm}$ for $5 \mathrm{~min}$ to obtain the supernatant. The concentrations of TNF- $\alpha$ and IL- 6 in the liver tissue were detected by ELISA according to the kit instructions.

2.6. Survival Rate. A 24-hour survival group comprising 21 rats was randomly divided into 3 groups: (1) control group, $n=7$; (2) disease model group, $n=7$; and (3) JDNW group, $n=7$. Survival rates were measured at $24 \mathrm{~h}$ after administration of combined HSA, LPS, and D-GalN. 
TABle 1: Composition of JDNW.

\begin{tabular}{lcccc}
\hline Components & Amount used $(\mathrm{g})$ & Botanical name of plant & Part used & Family \\
\hline Phyllanthus urinaria Linn. & 30 & Phyllanthus amarus Schumach. \& Thonn & Herb & Euphorbiaceae \\
Radix Astragali & 30 & Astragalus membranaceus (Fisch.) Bunge & Root & Leguminosae \\
Fructus Trichosanthis & 30 & Trichosanthes kirilowii Maxim & Fruit & Cucurbit \\
Herba Lysimachiae & 30 & Lysimachia christinae Hance & Herb & Primulaceae \\
Herba Visci & 30 & Viscum coloratum (Kom.) Nakai & Stem, leaf & Loranthaceae \\
Radix et Rhizoma Notoginseng & 6 & Panax notoginseng (Burkill) F. H. Chen & Root & Araliaceae \\
Rhizoma Curcumae & 6 & Curcuma phaeocaulis Valeton & Rhizome & Zingiberaceae \\
Salvia miltiorrhiza Bunge & 20 & Salvia miltiorrhiza Bunge & Root & Lamiaceae \\
Radix Rehmanniae & 20 & Rehmannia glutinosa (Gaertn.) DC & Root & Scrophulariaceae \\
Radix Aconiti Lateralis Preparata & 15 & Aconitum carmichaeli var. carmichaeli & Root & Ranunculaceae \\
\hline
\end{tabular}
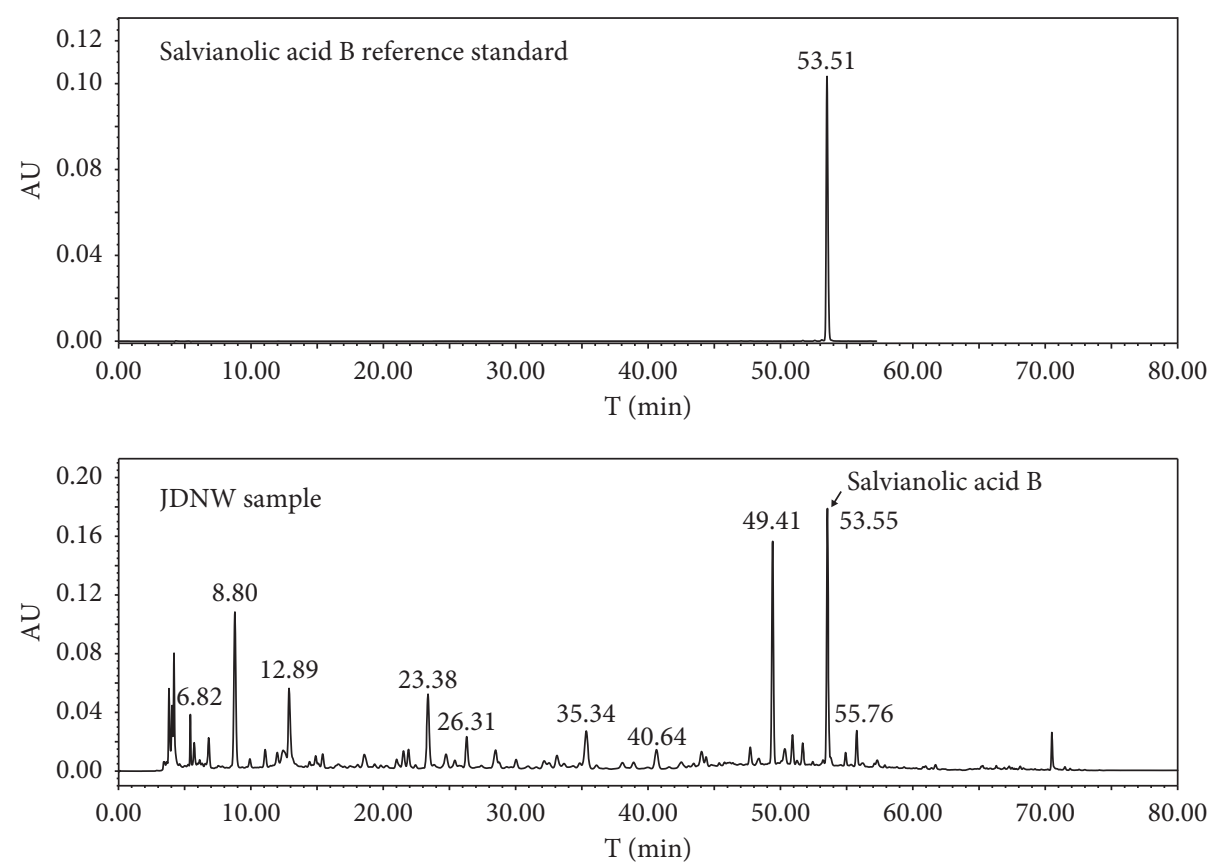

(a)<smiles>O=C(/C=C\c1ccc(O)c2c1OC(c1ccc(O)c(O)c1)C2C(=O)OC(Cc1ccc(O)c(O)c1)C(=O)O)OC(Cc1ccc(O)c(O)c1)C(=O)O</smiles>

(b)

FIgure 1: (a) The HPLC chromatograms of standard salvianolic acid B and JDNW extract. (b) Salvianolic acid B chemical structure.

2.7. Histological Observation of the Liver. Embedded liver tissues were sliced and stained with hematoxylin-eosin (HE). The stained samples were observed with a Nikon Eclipse $80 \mathrm{i}$ microscope at $\times 200$ magnification.

2.8. TUNEL Assays. TUNEL assay was performed according to the manufacturer's protocol (Beyotime). Apoptosis was observed with a Nikon Eclipse $80 \mathrm{i}$ microscope at $\times 200$ magnification. According to the distribution of apoptotic cells, 5 areas were analyzed per section, and 100 cells were counted in each area. The apoptotic index (AI) was calculated as the percentage of the number of apoptotic cells/total number of cells.

2.9. Transmission Electron Microscopy (TEM) Observation. Liver specimens were fixed with $2.5 \%$ glutaraldehyde for $2 \mathrm{~h}$ and washed three times for $10 \mathrm{~min}$ each with phosphate buffer. After dehydration with ethanol and propanol, liver 


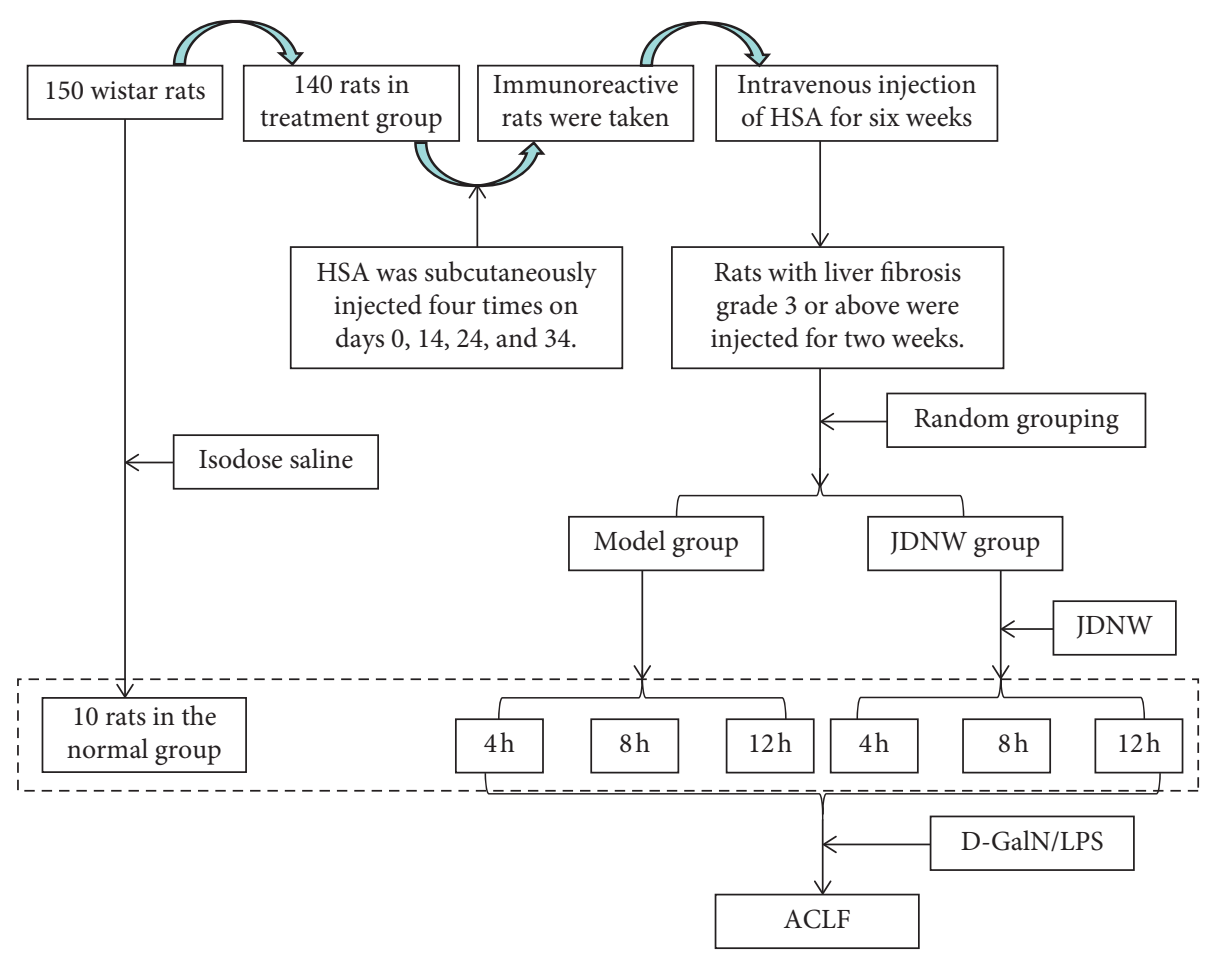

FIgURE 2: Experimental setup.

specimens were embedded in epoxy resin sliced and stained with uranium acetate and lead citrate. The ultrastructure of hepatocytes and mitochondria was observed and photographed by transmission electron microscopy.

2.10. Western Blot Analysis. Liver tissues (50-100 mg) were harvested and homogenized from 6 rats in each group. The protein concentration in each sample was determined according to the kit instructions. An aliquot of $30 \mu \mathrm{g}$ from each sample was separated by $12 \%$ SDS-PAGE for $1 \mathrm{~h}$ at $120 \mathrm{~V}$. Bands were electrophoretically transferred to $0.22 \mu \mathrm{m}$ PVDF membranes (Millipore, USA), then blocked for nonspecific binding with $5 \%$ nonfat dry milk for $1 \mathrm{~h}$, and incubated overnight at $4^{\circ} \mathrm{C}$ with primary antibodies. The next day, after washing, the membranes were incubated with Goat Anti-Mouse IgG and Goat Anti-Rabbit IgG (Santa Cruz Biotechnology, USA) for $1 \mathrm{~h}$ reaction, washed again, and developed with an electrochemiluminescence (ECL) reagent (Millipore, USA). Blots were developed by autoradiography. $\beta$-Actin antibody (Abcam, USA, $1: 3000$ ) was used as an internal control. Images were analyzed and quantified by using ImageJ software. The following primary antibodies were used: E2F-1 antibody (Santa Cruz Biotechnology, USA, 1:500), P53 antibody (Santa Cruz Biotechnology, USA, 1:1000), P73 antibody (Abcam, USA, 1 : 1000), Apaf-1 antibody (Santa Cruz Biotechnology, USA, 1 : 1000), caspase- 3 antibody (CST, USA, $1: 1000$ ), caspase-6 antibody (Proteintech, 1:500), caspase-7 antibody (Santa, USA, $1: 1000$ ), p14ARF antibody (Abcam, USA, $1: 500$ ), Bcl2 antibody (Santa Cruz Biotechnology, USA, 1:1000), and Mcl-1 antibody (Abcam, USA, $1: 1000$ ).
2.11. PCR. Total RNA was extracted from rat liver samples using TRIzol reagent and its concentration and purity were determined using a spectrophotometer. RNA samples with A260/A280 from 1.8 to 2.2 were selected and their integrity was tested on $2 \%$ agarose gels. The total RNA was then reverse-transcribed into cDNA using PrimeScript ${ }^{\mathrm{TM}}$ RT reagent Kit with gDNA Eraser. PCR primers were designed and synthesized by Invitrogen; the primer sequences have been provided in Supplementary Material (available here). Finally, real-time PCR was performed on target genes and the data were analyzed by $2^{-\Delta \Delta \mathrm{Ct}}$ method.

2.12. Statistical Analyses. Statistical analyses were performed by using SPSS software version 19.0. Experiments were repeated at least three times and results presented as mean \pm standard deviation (SD). One-way ANOVA was used to compare the significance of the differences among groups, the least-significant difference (LSD) method was applied when the variance was equal among groups, and Tamhane's T2 test was performed otherwise. $P$ values $<0.05$ were considered to be statistically significant.

\section{Results}

3.1. Serum ALT, AST, and TBIL Levels and Survival Analysis. Figure 3(a) shows the analysis of liver function parameters. Compared to the control group, ALT, AST, and TBIL levels in the ACLF model group were significantly and continuously increased through time $(P<0.01)$, which indicated the presence of severe liver damage. However, the JDNW group showed reduced ALT, AST, and TBIL serum levels. 

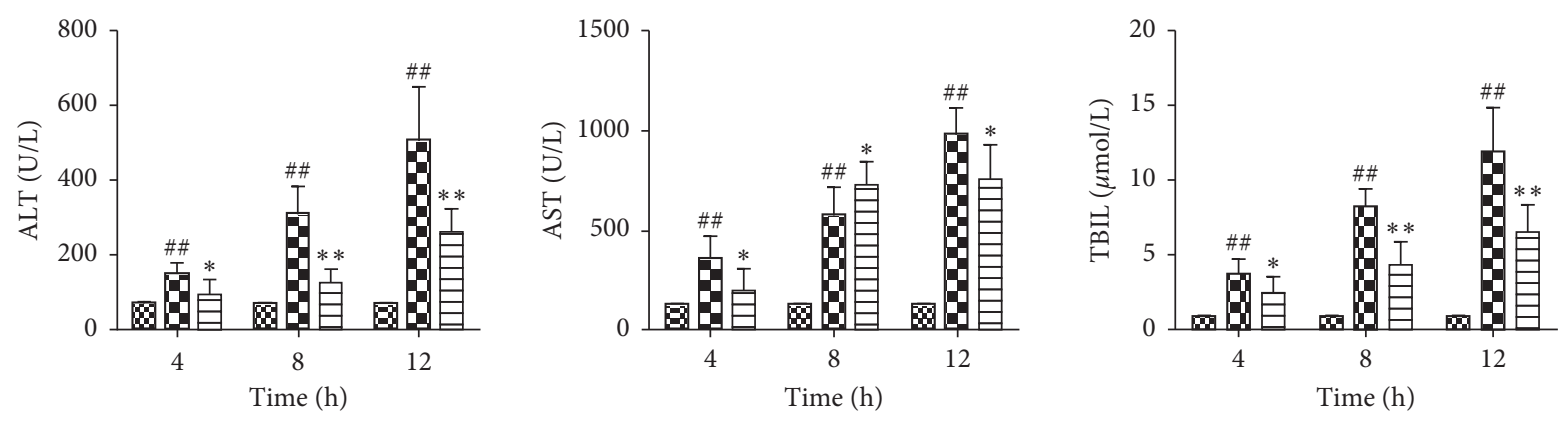

$$
\begin{aligned}
& \text { (20) Control } \\
& \text { 모 Model } \\
& \text { 曰 JDNW }
\end{aligned}
$$

$$
\begin{aligned}
& \text { ‥ Control } \\
& \square \text { Model } \\
& \square \text { JDNW }
\end{aligned}
$$

(10) Control

B. Model

$\boxminus$ JDNW

(a)
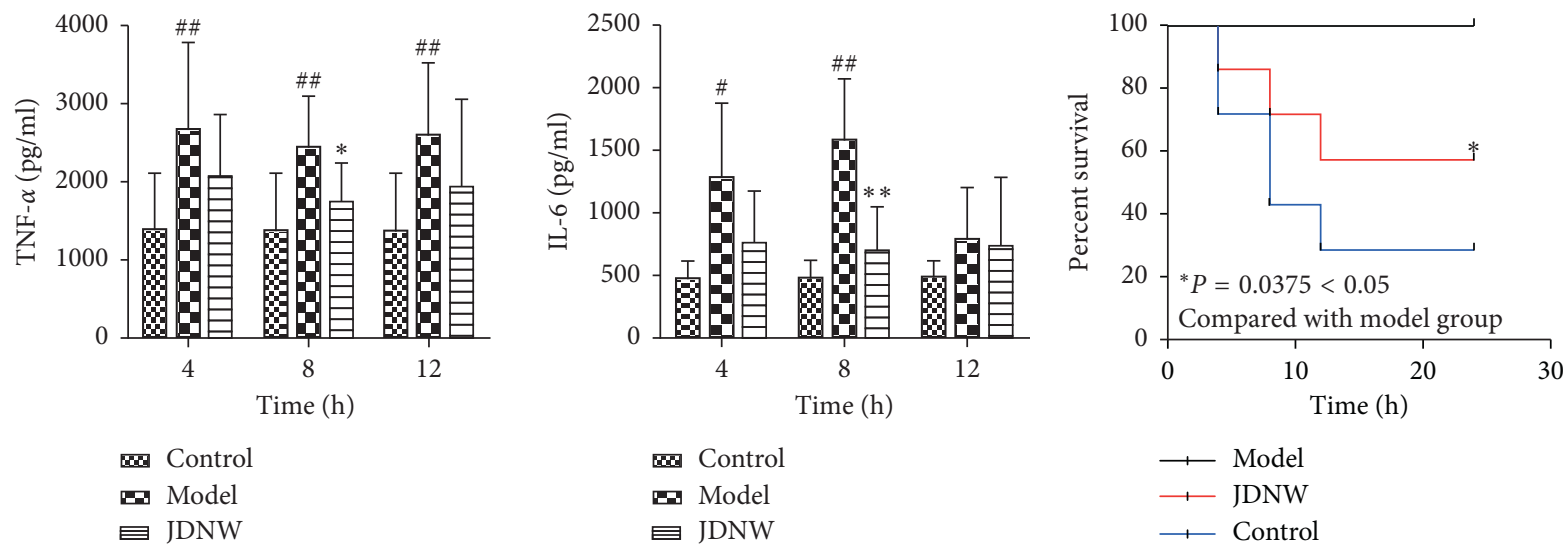

(b)

(c)

FIGURE 3: Rat liver functions, assessed by levels of parameters, inflammatory factors, and survival rates. (a) Levels of ALT, AST, and TBIL. (b) Levels of TNF- $\alpha$ and IL-6. (c) ACLF rat survival curves within 24 hours. ${ }^{\#} P<0.05$; ${ }^{\# \#} P<0.01$, versus control group. ${ }^{*} P<0.05$; ${ }^{* *} P<0.01$, versus model group.

Differences were statistically significant at 8 and $12 \mathrm{~h}$ $(P<0.01)$. The Kaplan-Meier survival curves (Figure 3(c)) showed that the 24-hour survival rate of JDNW group was significantly increased compared to that of the ACLF model group $(P<0.05)$. After 24 hours, only 2 rats survived (survival rate $=28 \%$ ) in the model group, whereas 4 rats survived (survival rate $=57 \%$ ) in the JDNW group. Overall, the survival time of the JDNW group was longer than that of the model group $(P<0.05)$ (Table 2$)$.

3.2. ELISA. Levels of TNF- $\alpha$ and IL-6 proteins (Figure 3(b)) were remarkably increased in the ACLF model group; however, treatment with JDNW significantly reduced their levels at 8 h (TNF- $\alpha$ : $P<0.05$; IL-6: $P<0.01)$.

3.3. Liver Histology Observation. A representative picture of HE-stained liver tissue for each group is shown in Figures 4(a) and 4(b). In the control group, liver cells were arrayed around the central vein and neither degeneration nor necrosis was presented. In the ACLF model group, hepatocyte swelling, apoptotic body formation, inflammatory cell infiltration, internal bleeding, disorganized liver cell arrangement, and massive or submassive necrosis
TABLE 2: Comparison of survival time of rats in each group.

\begin{tabular}{llc}
\hline Groups & N & Survival periods $(\mathrm{h})$ \\
\hline Model & 7 & $12.00 \pm 8.64$ \\
JDNW & 7 & $17.14 \pm 8.86^{*}$ \\
\hline
\end{tabular}

${ }^{*} P<0.05$ versus model group.

were revealed in some areas and the severity increased over time. However, the histological characteristics of the liver were significantly improved at each corresponding time point in the JDNW group.

3.4. TUNEL Assay Results. As shown in Figure 4(c), apoptotic cells were detected in the control group. On the contrary, a large number of apoptotic cells with brown nucleus appeared at 4,8 , and $12 \mathrm{~h}$ and gradually increased over time in the model group. However, apoptotic cells in the JDNW group decreased at the corresponding time points. These results suggested that JDNW could alleviate hepatocyte apoptosis and reduce the apoptotic index.

3.5. Ultrastructure Observation. In the control group, the hepatocyte membrane (Figure 5(a)) was intact, the 


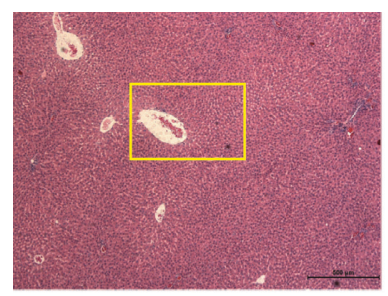

Control

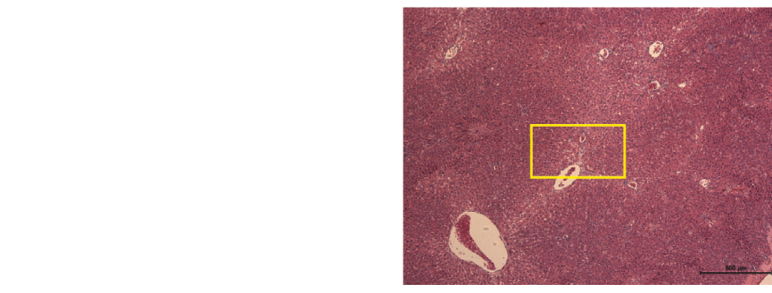

JDNW at $4 \mathrm{~h}$

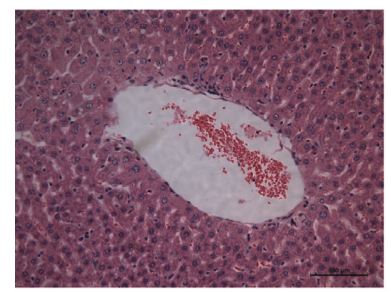

Control

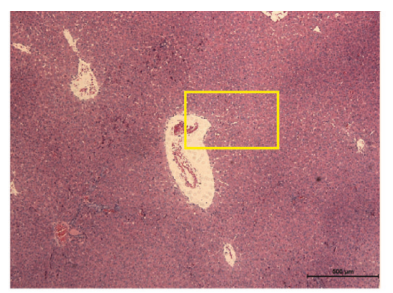

Model at 4h

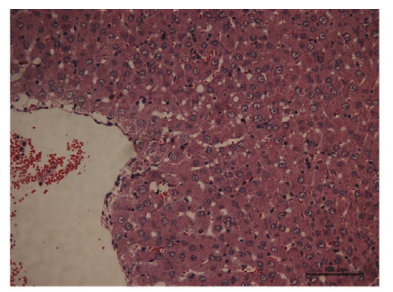

Model at $4 \mathrm{~h}$

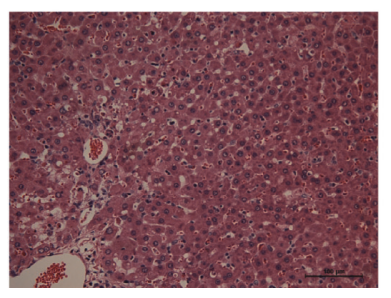

JDNW at $4 \mathrm{~h}$

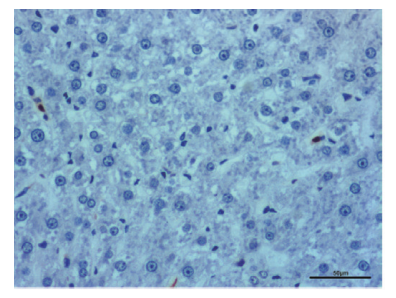

Control

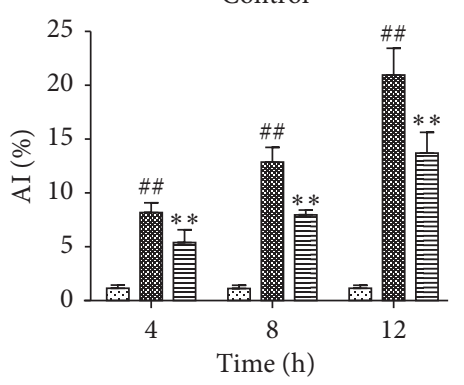

Control
Model
․․․․

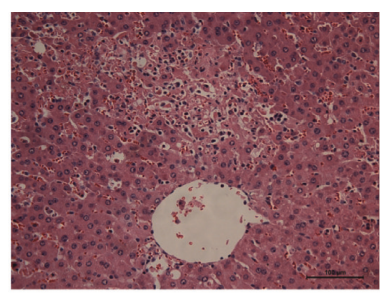

Model at $8 \mathrm{~h}$

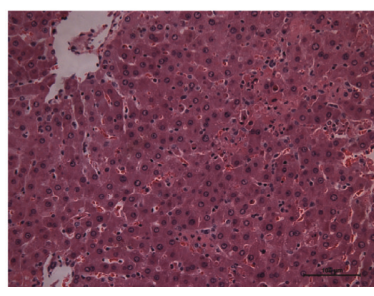

JDNW at $8 \mathrm{~h}$

(b)

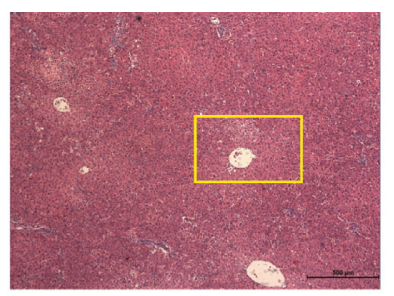

Model at $8 \mathrm{~h}$

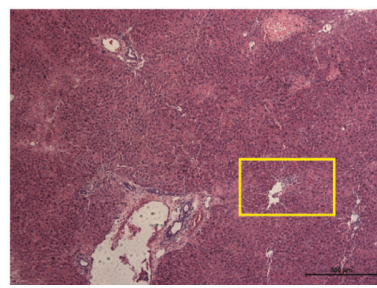

JDNW at $8 \mathrm{~h}$

(a)

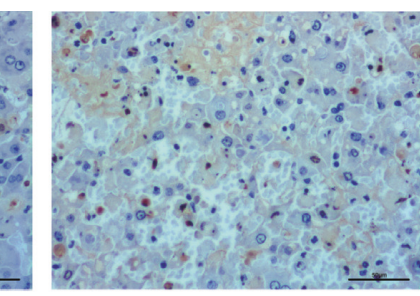

Model at $8 \mathrm{~h}$

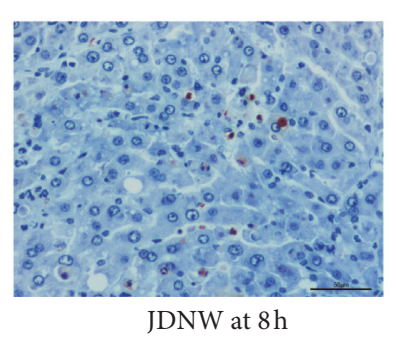

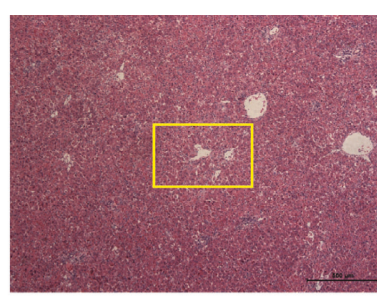

Model at $12 \mathrm{~h}$

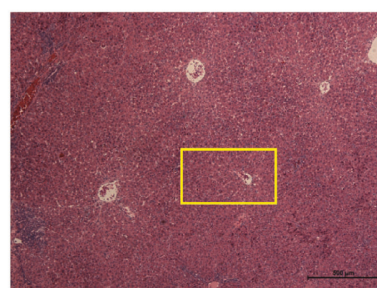

JDNW at $12 \mathrm{~h}$

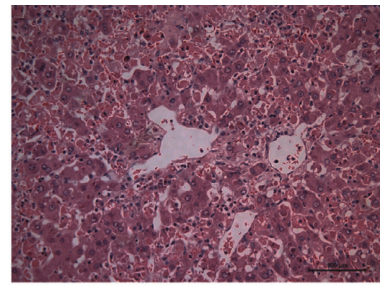

Model at $12 \mathrm{~h}$

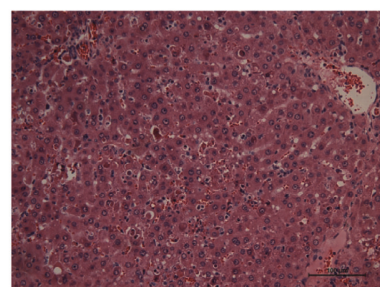

JDNW at $12 \mathrm{~h}$

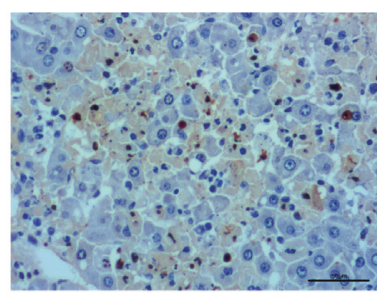

Model at $12 \mathrm{~h}$

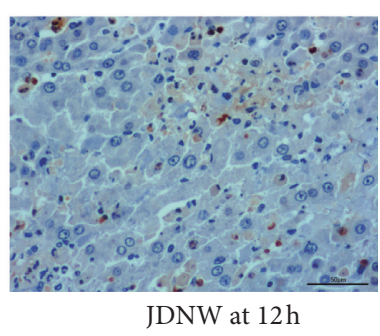

(c)

FIgURE 4: The histological changes in each group were observed after HE staining. (a) Magnification $\times 50$. (b) Magnification $\times 200$. (c) TUNEL assay results at different time points. ${ }^{\#} P<0.05 ;{ }^{\#} P<0.01$, versus control group. ${ }^{*} P<0.05 ;{ }^{* *} P<0.01$, versus model group. 


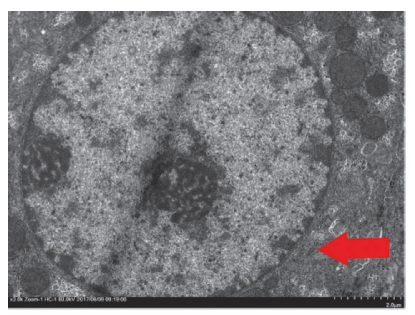

Control

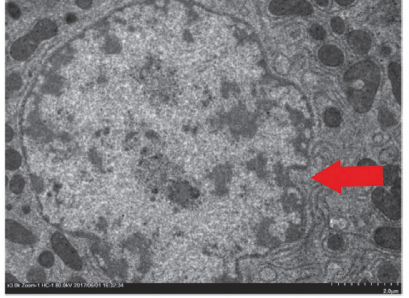

Model at $4 \mathrm{~h}$

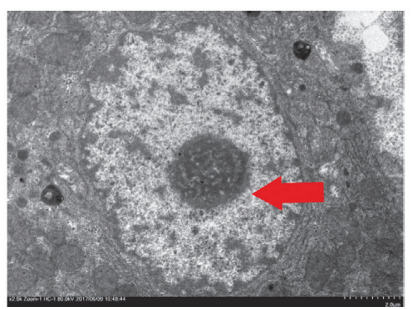

JDNW at $4 \mathrm{~h}$

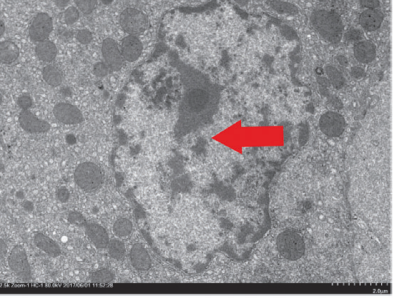

Model at $8 \mathrm{~h}$

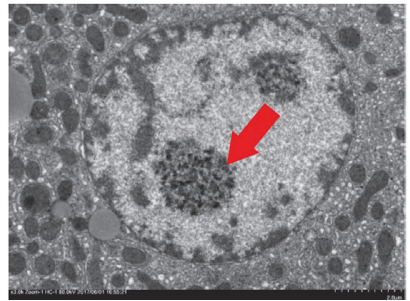

JDNW at $8 \mathrm{~h}$

(a)

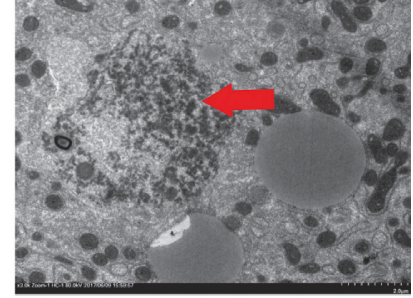

Model at $12 \mathrm{~h}$

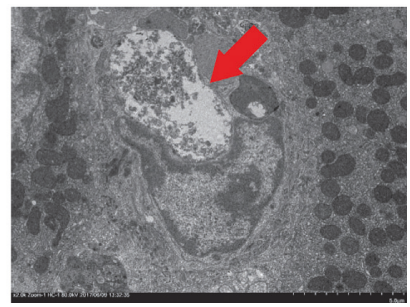

JDNW at $12 \mathrm{~h}$

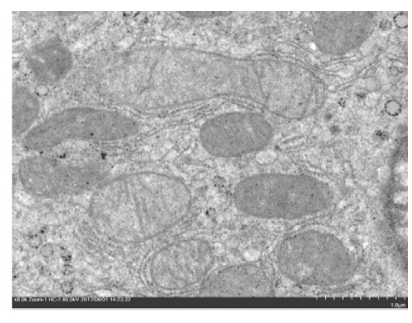

Model at $12 \mathrm{~h}$

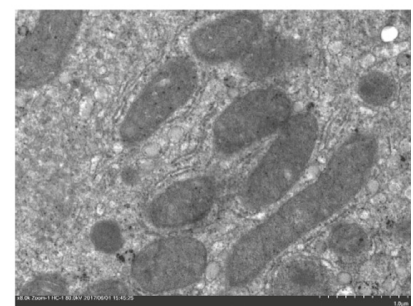

JDNW at $12 \mathrm{~h}$

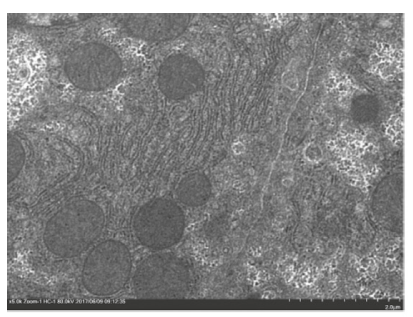

Control

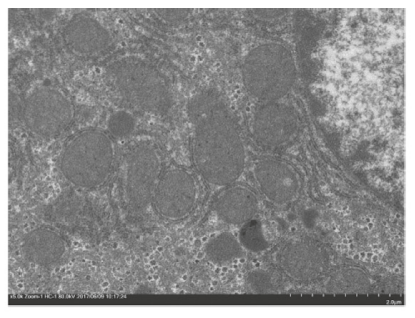

Model at $4 \mathrm{~h}$

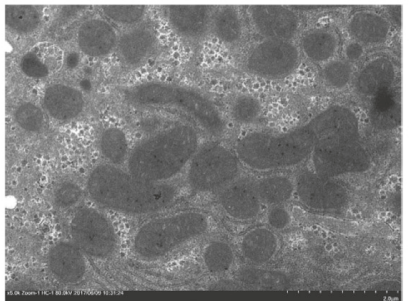

JDNW at $4 \mathrm{~h}$

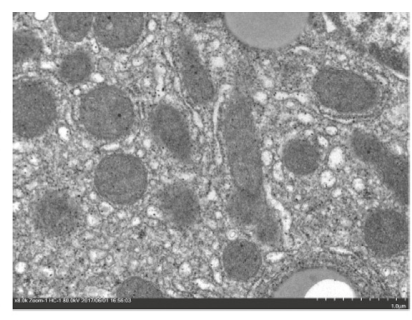

Model at 8h

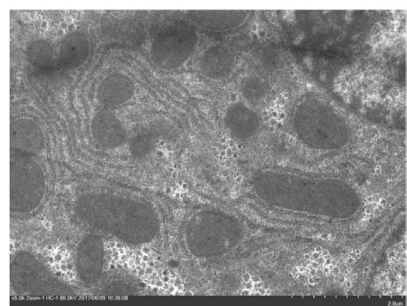

JDNW at $8 \mathrm{~h}$

(b)

FIGURE 5: (a) Ultrastructure changes of rat hepatocytes in the rats of each group (bar $=2 \mu \mathrm{m}$ ). (b) Ultrastructure changes of mitochondria in the rat hepatocytes of the rats in each group (bar $=500 \mathrm{~nm})$.

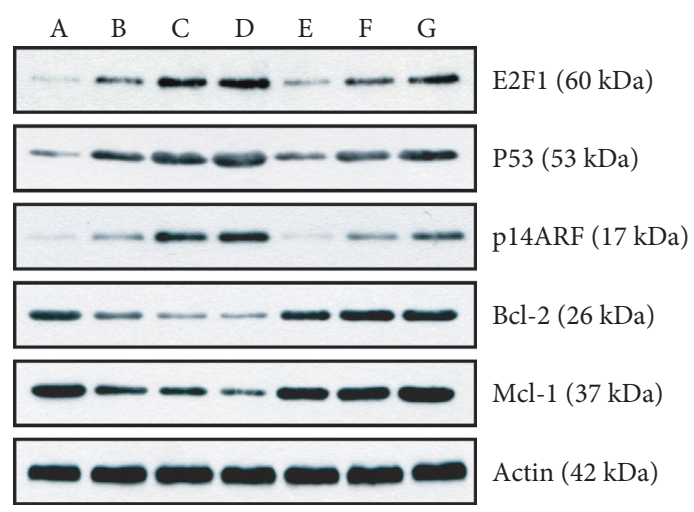

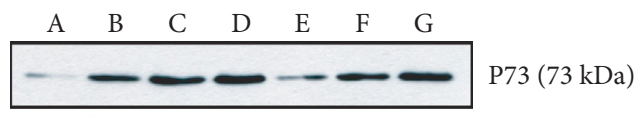

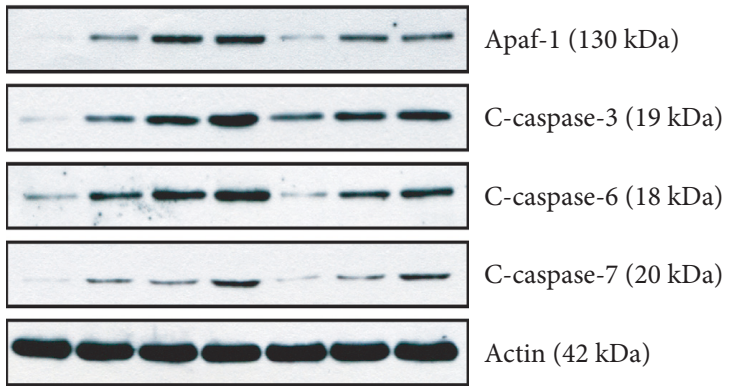

(a)

Figure 6: Continued. 

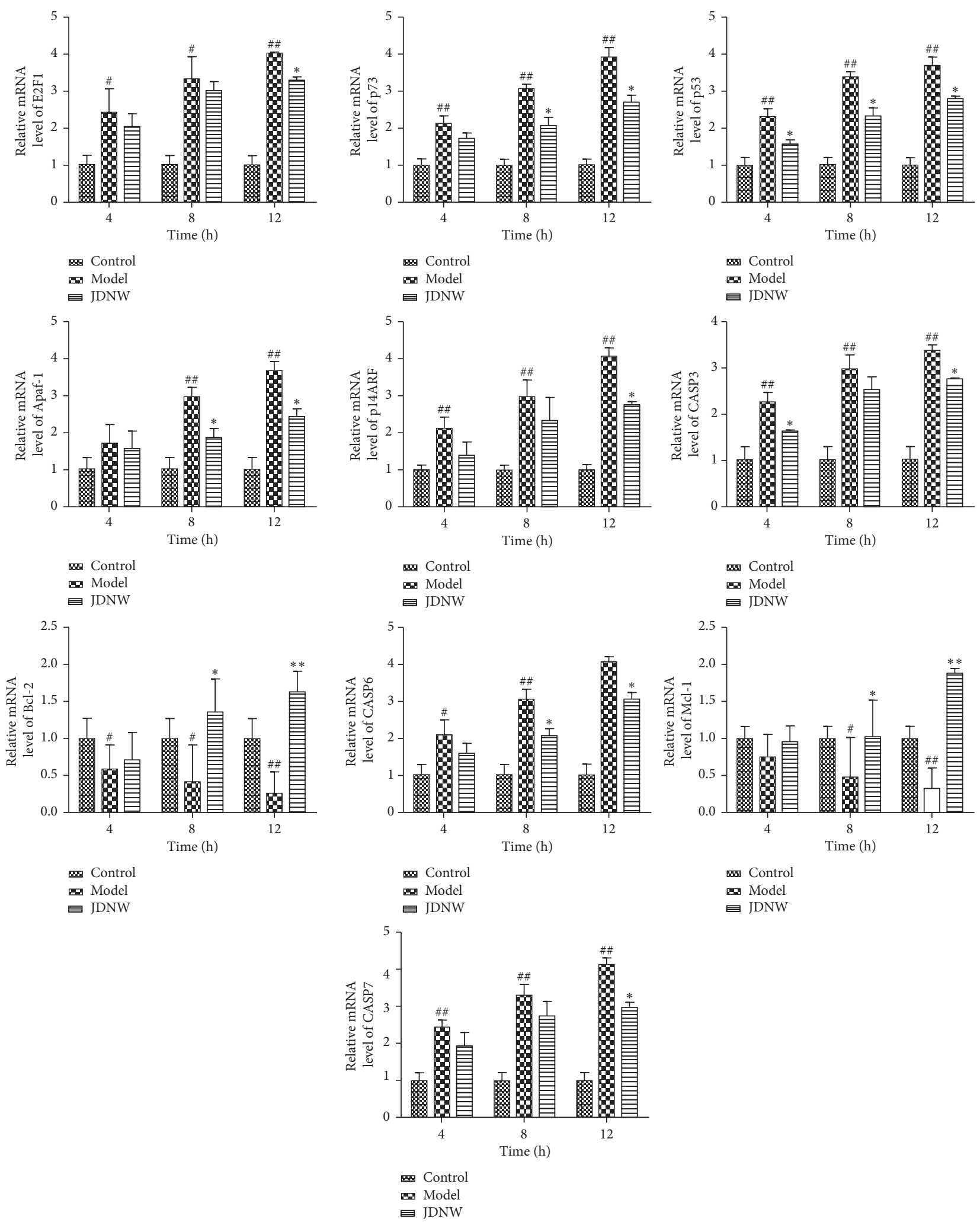

Control
$\square$ Model
$\square$ JDNW

(b)

FIGURE 6: Effect of JDNW on expression of E2F1, P53, P73, p14ARF, Apaf-1, caspase-3, caspase-6, caspase-7, Bcl-2, and Mcl-1 levels in liver tissues of ACLF model rats. (a) Western blot analysis. A: control. B: model at $4 \mathrm{~h}$. C: model at $8 \mathrm{~h}$. D: model at $12 \mathrm{~h}$. E: JDNW at $4 \mathrm{~h}$. F: JDNW at 8 h. G: JDNW at 12 h. (b) mRNA expression. ${ }^{\#} P<0.05 ;{ }^{\# \#} P<0.01$, versus control group. ${ }^{*} P<0.05 ;{ }^{* *} P<0.01$, versus model group. 
cytoplasm was homogeneous, and the nucleus was round and centered. The shape of mitochondria (Figure 5(b)) was round, and the structures of the cristae and bilayer membrane were intact. In the 4-hour model group, nuclear deformation and chromatin condensation were observed. Also, cytoplasm became gradually empty; meanwhile, mitochondria presented a swelling, dumbbell appearance. In the 12-hour model group, irregular or ruptured hepatocytes nuclei were observed; meanwhile, Kupffer cells invaded necrotic cells and engulfed apoptotic bodies. Comparing with the model group, the ultrastructures of hepatocytes and mitochondria in the JDNW group were significantly improved at each corresponding time point.

3.6. Western Blotting and PCR Analysis. As compared with the control group, expression levels of E2F1, P53, p14ARF, P73, Apaf-1, and cleaved caspase-3, caspase-6, and caspase-7 were increased, whereas those of $\mathrm{Bcl}-2$ and $\mathrm{Mcl}-1$ were decreased in the ACLF model group (Figures 6(a) and 6(b)). Treatment with JDNW reduced E2F1, P53, p14ARF, P73, Apaf-1, and cleaved caspase- 3 , caspase- 6 , and caspase-7 levels but increased Bcl-2 and Mcl-1 levels (protein expression data have been provided in the supplementary data of this manuscript).

\section{Discussion}

ACLF is a syndrome characterized by acute jaundice and coagulation dysfunction. It can be combined with complications such as hepatic encephalopathy, ascites, electrolyte disturbance, infection, hepatorenal syndrome, hepatopulmonary syndrome, extrahepatic organ failure, and new hepatocyte necrosis lesions of varying degrees [18]. Administration of LPS and D-GalN is a commonly used approach to generate a rat model of ALF [19]; however, only two different methods have been used to induce the basic chronic liver injury: $\mathrm{CCl}_{4}$ and HSA [20-22]. When compared with the hepatotoxic drug $\mathrm{CCl}_{4}$, the immunologic stimulant HSA can induce a more stable model with lower healing rate, and a pathogenetic mechanism more similar to that in ACLF $[23,24]$. Therefore, we used a combined administration of HAS, LPS, and D-GalN to generate the animal model in the study.

Disturbance and necrosis of hepatocytes and elevated levels of ASL and ALT suggested liver failure [25]. Our results showed that, ALT, AST, and TBIL protein levels increased in the ACLF model group. In addition, histopathology analysis showed massive or submassive necrosis and collapse of parenchymal, TEM examination detected nuclear fragmentation, vacuolization of mitochondria, and apoptotic bodies formation, which indicated that the ACLF model was successfully established. After JDNW intervention, liver function improved, the apoptotic index decreased, and survival times were prolonged.

JDNW, originally created by Qian Ying professor, has been used in Beijing You'an Hospital, Capital Medical University, for many years. Previous clinical trials have also confirmed the protective effects of JDNW on ACLF patients
[7]. The drug contained ten Chinese herb medicines, among them, Phyllanthus urinaria Linn., Fructus Trichosanthis, Herba Lysimachiae, Radix et Rhizoma Notoginseng alleviated heat, induced disintoxication, resolved stasis, and eliminated jaundice. Also, Radix Astragali and Herba Visci played the role of tonifying spleen and nourishing the liver and kidney. Rhizoma Curcumae and Salvia miltiorrhiza Bunge played the role of resolving toxin and dredging collaterals. Radix Rehmanniae and Radix Aconiti Lateralis Preparata regulated Yin and Yang.

However, abstract TCM theory is not able to fully explain the mechanism of JDNW in treating liver failure. LPSand D-GalN-induced liver failure is associated with oxidative stress, inflammatory response, and apoptosis [26]. E2F1 is an activator of transcription and has the function of regulating apoptosis and proliferation [27]. In addition, E2F1 had the ability to induce apoptosis in many cells $[28,29,30,31]$. Based on this, we aimed to study whether JDNW regulates E2F1 pathway. In the p53-dependent pathway, E2F-1 expression induced apoptosis through the accumulation of p53 and thus by activating p14ARF expression [32]. In our study, E2F1, p53, and p14ARF levels gradually increased in the model group but decreased in the JDNW group. The results suggested that transcription factors were activated, and JDNW had an antiapoptotic effect. In the p53-independent apoptosis pathway, E2F1 directly activated some apoptotic genes, such as p73, the homologous gene of p53 [33], and Apoptotic protease activating factor 1 (Apaf-1) [34], thereby activating the proapoptotic effector enzymes caspase- 3 , caspase- 6 , and caspase- 7 and inducing apoptosis [35]. Protein expression of P73 and Apaf- 1 in the model group, and of their downstream genes, caspase-3, caspase-6, and caspase-7 increased in our study. When apoptosis occurs, caspase- 3 , caspase- 6 , and caspase- 7 in the proenzyme forms are cleaved into cleaved caspase-3, caspase- 6 , and caspase- 7 , respectively. Apaf- 1 is the core component of apoptosis body and plays an important role in the mitochondrial apoptotic pathway [36]. Besides protecting mitochondrial morphology, JDNW also reduced the expression of P73 and Apaf- 1 and inhibited the activation of caspase- 3 , caspase- 6 , and caspase- 7 and thus inhibited apoptosis. E2F1 also blocks antiapoptotic pathway and induces apoptosis by inhibiting Bcl-2 and Mcl-1 [37], which are antiapoptotic members of the Bcl-2 family. E2F-1 inhibited Bcl-2 and Mcl-1 expression and induced apoptosis in the model group. Compared to the model group, $\mathrm{Bcl}-2$ and $\mathrm{Mcl}-$ 1 expression increased in the JDNW group at corresponding time points, which suggested that JDNW exerted an antiapoptotic effect.

Serum TNF- $\alpha$ and IL- 6 are more sensitive than ALT and AST levels in predicting liver injury [30]. TNF- $\alpha$ is a primary and central inflammatory cytokine that induces hepatocyte apoptosis and liver injury [31]. IL-6 plays a role as a proinflammatory cytokine in chronic inflammatory disease models $[38,39]$. Additionally, acute inflammatory IL-6 driven response might contribute to the transition from a stable chronic state to a progressive liver injury [40]. Following treatment with JDNW, the expression of inflammatory cytokines was inhibited. Therefore, the effect of 


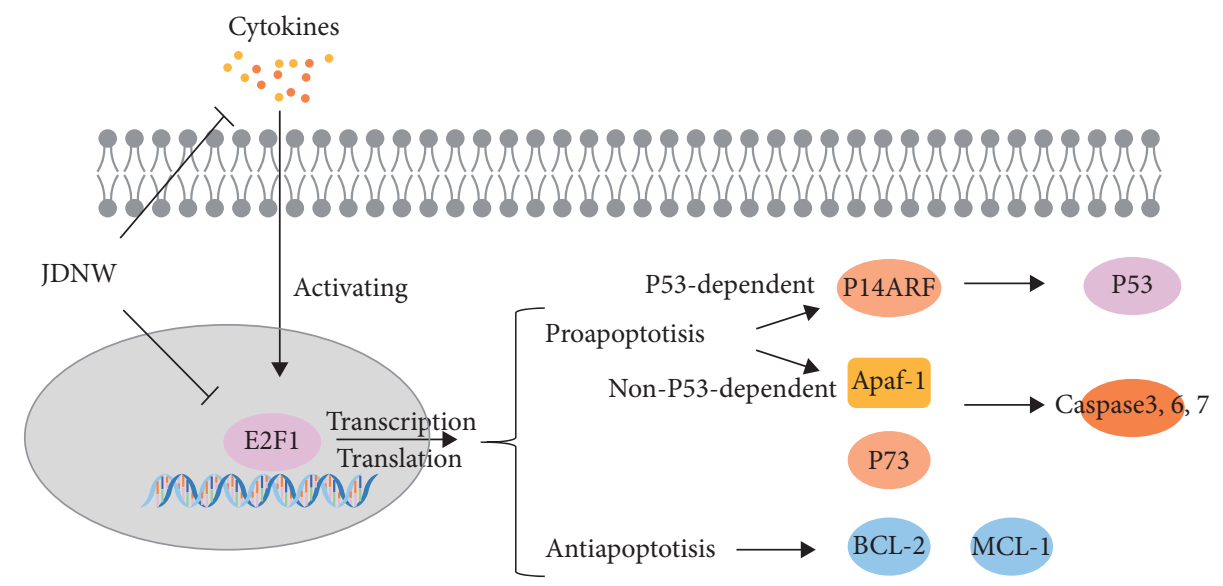

FIgURE 7: The mechanism of E2F1-induced apoptosis, and inhibition of this pathway by JDNW in ACLF rats.

ACLF on the E2F1 mediated intrinsic apoptosis pathway was mainly due to the in vivo increase of endotoxins, which promoted the secretion of inflammatory cytokines, such as TNF- $\alpha$ and IL-6, causing apoptosis.

ACLF rat model was successfully reproduced and our results suggested that JDNW could inhibit liver failure by inhibiting hepatocyte apoptosis. However, the time required to induce ACLF is longer and the process is more complex. Therefore, simpler methods need to be urgently explored. Moreover, there are too many targets of TCM; whether JDNW alone acts on E2F1 and then changes downstream proteins remains to be confirmed. Network pharmacology has advantages in analyzing the relationship between Chinese herbal compound and target [41]. Therefore, the next step is to combine network pharmacology with in vitro experiments and to add E2F1 inhibitors to confirm the role of JDNW.

\section{Conclusions}

In summary, this study suggested that JDNW could significantly protect rat liver and attenuate hepatocyte apoptosis in ACLF rats. We also showed that this effect was due to inhibition of the E2F1-mediated intrinsic apoptosis pathway. Therefore, we propose a therapeutic pathway through which JDNW could reduce liver apoptosis in ACLF (Figure 7).

\section{Data Availability}

The data used to support the findings of this study are available from the corresponding author upon request.

\section{Conflicts of Interest}

The authors declare that they have no conflicts of interest.

\section{Acknowledgments}

This work was supported by the National Natural Science Foundation of China (Grant no. 81573767, awarded to Qiuyun Zhang).

\section{Supplementary Materials}

(1) Masson staining of cirrhosis. (2) Primer sequence used for real-time PCR. (3) Protein expression data for each protein. (Supplementary Materials)

\section{References}

[1] L. Yang, T. Wu, J. Li, and J. Li, "Bacterial infections in acuteon-chronic liver failure," Seminars in Liver Disease, vol. 38, no. 2, pp. 121-133, 2018.

[2] R.-H. Zhao, Y. Shi, H. Zhao, W. Wu, and J.-F. Sheng, "Acuteon-chronic liver failure in chronic hepatitis B: an update," Expert Review of Gastroenterology \& Hepatology, vol. 12, no. 4, pp. 341-350, 2018.

[3] R. Hernaez, E. Solà, R. Moreau, and P. Ginès, "Acute-onchronic liver failure: an update," Gut, vol. 66, no. 3, pp. 541-553, 2017.

[4] A. Azeem, K. C. Suen, and D. Ma, "Acute-on-chronic liver failure: recent update," The Journal of Biomedical Research, vol. 31, no. 4, pp. 283-300, 2016.

[5] L. Wang, L. I. Jun, L. I. Fengyi et al., "A multicentered, randomize-controlled trial of integrative medicine for acuteon-chronic (subacute) liver failure," Journal of Traditional Chinese Medicine, vol. 54, no. 22, pp. 1922-1925, 2013.

[6] J. H. Hu, Y. Qian, N. L. Yao et al., "Treatment of chronic severe hepatitis B by Jieduan Niwan method," Chinese Journal of Integrated Traditional \& Western Medicine on Liver Diseases, vol. 20, no. 4, pp. 200-203, 2010.

[7] F. Li, L. Miao, H. Sun, Y. Zhang, X. Bao, and D. Zhang, "Establishment of a new acute-on-chronic liver failure model," Acta Pharmaceutica Sinica B, vol. 7, no. 3, pp. 326333, 2017.

[8] Z. Dang, L. I. Jinxia, Q. Zhang et al., "Effects of truncation and inversion prescription on the ultrastructure, Cyclin E and its transcription factor DP-1 in rats with ACLF," Hebei Medical Journal, vol. 9, pp. 1285-1289, 2015.

[9] J.-X. Li, L.-Y. Mu, Q. Y Zhang, Y. Chen, L. Gao, and Y. Du, "Effects of Jieduan Niwan formula on mitochondrial apoptotic pathway in acute-on-chronic liver failure rats," Chinese Journal of Information on Traditional Chinese Medicine, vol. 23, no. 4, pp. 45-48, 2016.

[10] L.-Y. Mu, J.-X. Li, Q. Zhang et al., "Effect of Jieduan Niwan prescription on death receptor pathway in acute-on-chronic 
liver failure rat model," Global Traditional Chinese Medicine, vol. 10, no. 4, pp. 410-415, 2017.

[11] L.-Y. Mu, J.-X. Li, Q. Y. Zhang et al., "Effects of Jieduan Niwan formula on the c-Jun,p-JNK of liver tissue in acute-onchronic liver failure rats," Journal of Capital Medical University, vol. 38, no. 2, pp. 282-288, 2017.

[12] Y. Hao, W. Yang, W. Hou et al., "Regulation mechanism of Jieduan Niwan formula on E2F1 signaling pathway in rats with acute-on-chronic hepatic failure," Global Traditional Chinese Medicine, vol. 11, no. 3, pp. 321-326, 2018.

[13] W. X. Hou, Y. L. Hao, W. L. Yang et al., "Effects of Jieduan Niwan prescription on IL- 6 and TNF- $\alpha$ in serum and hepatic tissue of acute-on-chronic liver failure rats," Chinese Journal of Information on Traditional Chinese Medicine, vol. 25, no. 07, pp. 49-52, 2018.

[14] Y. Hao, W. Yang, W. Hou et al., "Effects of Jieduan Niwan formula on the pathway of cell proliferation in ACLF rats," Chinese Journal of Information on Traditional Chinese Medicine, vol. 35, no. 3, pp. 12-17, 2018.

[15] H. Yulin, Y. Wenlong, H. Weixin et al., "Regulation mechanism of Jieduan Niwan formula on E2F1 signaling pathway in rats with acute-on-chronic hepatic failure," Global Traditional Chinese Medicine, vol. 11, no. 3, pp. 321-326, 2018.

[16] X. H. Liu, Y. Chen, T. L. Wang et al., "Establishment of a D-galactosamine/lipopolysaccharide induced acute-onchronic liver failure model in rats," Chinese Journal of Hepatology, vol. 15, no. 10, p. 771, 2007.

[17] Z. L. Liu, W. X. Wu, and Q. Y. Zhang, "The influence of the prescription of truncation and inverse draft on the ET and TNF- $\alpha$ of acute-on-chronic liver failure model rats," Chinese Journal of Medicinal Guide, vol. 13, no. 4, pp. 650-652, 2011.

[18] Liver failure and Artificial Liver Group, Chinese Society of Infectious Diseases, Chinese Medical Association and Severe Liver Diseases and Artificial Liver Group, Chinese Society of Hepatology, Chinese Medical Association, "Guideline for the diagnosis and treatment of liver failure (2018 edition)," Chinese Journal of Clinical Infectious Diseases, vol. 11, no. 6, pp. 401-409, 2018.

[19] H. Takano, M. Satoh, and K.-I. Inoue, "Protective role of metallothionein in coagulatory disturbance accompanied by acute liver injury induced by LPS/D-GalN," Thrombosis and Haemostasis, vol. 99, no. 11, pp. 980-983, 2008.

[20] X. Liu, Y. Chen, J. Zhang et al., "Human serum albumin and a rat's model for D-Galactosamine/Lipopolysaccharide induced acute-on-chronic liver failure," Science \& Technology Review, vol. 25, no. 18, pp. 32-37, 2007.

[21] H. Zhang, J. Lou, and N. Li, "Establishing of CCl 4-induced liver injury model in mice," Beijing Medical Journal, vol. 22, no. 3, pp. 199-202, 2011.

[22] H. Zhang, W. Hou, J. Liu et al., "The approaches for making acute hepatic failure model on chronic hepatic damage in rat," Chinese Journal of Gastroenterology and Hepatology, vol. 18, no. 4, pp. 348-351, 2009.

[23] P. Li, B. Liang, A. Wang et al., "Research on liver fibrosis rat model induced by CCl 4 and HSA," Laboratory Animal Science and Administrator, vol. 17, no. 2, pp. 32-34, 2000.

[24] X. Liu, Y. Meng, Y. Chen et al., "Exploration of establishing rat model of ACLF in human serum albumin-induced cirrhosis," Chinese Journal of Gastroenterology \& Hepatology, vol. 17, no. 10, pp. 791-794, 2008.

[25] J. Bae, Y. S. Min, Y. Nam, H. S. Lee, and U. D. Sohn, "Humulus japonicus extracts protect against lipopolysaccharide/d-galactosamine-induced acute liver injury in rats," Journal of Medicinal Food, vol. 21, no. 10, pp. 1009-1015, 2018.
[26] X. Gong, Y. Yang, L. Huang et al., “Antioxidation, anti-inflammation and anti-apoptosis by paeonol in LPS/D-GalNinduced acute liver failure in mice," International Immunopharmacology, vol. 46, pp. 124-132, 2017.

[27] H. Gao, X. Song, T. Kang et al., "Long noncoding RNA CRNDE functions as a competing endogenous RNA to promote metastasis and oxaliplatin resistance by sponging miR-136 in colorectal cancer," OncoTargets and Therapy, vol. 10, pp. 205-216, 2017.

[28] J. Gu, Y.-Q. Fan, H.-L. Zhang et al., "Resveratrol suppresses doxorubicin-induced cardiotoxicity by disrupting E2F1 mediated autophagy inhibition and apoptosis promotion," Biochemical Pharmacology, vol. 150, pp. 202-213, 2018.

[29] Z. Zhang, W. Liu, L. Zhao et al., "Retinoblastoma 1 protects $\mathrm{T}$ cell maturation from premature apoptosis by inhibiting E2F1," Development, vol. 145, no. 1, Article ID dev158139, 2018.

[30] G. Gao, Z. Yu, J. Yan et al., "Lowering blood ammonia prevents hepatocyte injury and apoptosis," International Journal of Clinical and Experimental Medicine, vol. 8, no. 8, pp. 12347-12355, 2015.

[31] D. S. Yu, F. M. An, B. D. Gong et al., "The regulatory role of microRNA-1187 in TNF- $\alpha$-mediated hepatocyte apoptosis in acute liver failure," International Journal of Molecular Medicine, vol. 29, no. 4, pp. 663-668, 2012.

[32] Y.-X. Zhao, H.-C. Liu, W.-Y. Ying et al., "microRNA-372 inhibits proliferation and induces apoptosis in human breast cancer cells by directly targeting E2F1," Molecular Medicine Reports, vol. 16, no. 6, pp. 8069-8075, 2017.

[33] R. M. Ramalho, P. S. Ribeiro, S. Solá et al., "Inhibition of the E2F-1/p53/bax pathway by tauroursodeoxycholic acid in amyloid $\beta$-peptide-induced apoptosis of PC12 Cells," Journal of Neurochemistry, vol. 90, no. 3, pp. 567-575, 2004.

[34] M. Irwin, M. C. Marin, A. C. Phillips et al., "Role for the p53 homologue p73 in E2F-1-induced apoptosis," Nature, vol. 407, no. 6804, pp. 645-648, 2000.

[35] M. C. Moroni, E. S. Hickman, E. L. Denchi et al., "Apaf-1 is a transcriptional target for E2F and p53," Nature Cell Biology, vol. 3, no. 6, pp. 552-558, 2001.

[36] M. Sancho, A. Gortat, A. E. Herrera et al., "Altered mitochondria morphology and cell metabolism in Apaf1-deficient cells," PLoS One, vol. 9, no. 1, Article ID e84666, 2014.

[37] R. Croxton, Y. Ma, L. Song, E. B. Haura, and W. D. Cress, "Direct repression of the Mcl-1 promoter by E2F1," Oncogene, vol. 21, no. 9, pp. 1359-1369, 2002.

[38] T. Alonzi, E. Fattori, D. Lazzaro et al., "Interleukin 6 is required for the development of collagen-induced arthritis," The Journal of Experimental Medicine, vol. 187, no. 4, pp. 461-468, 1998.

[39] M. Yamamoto, K. Yoshizaki, T. Kishimoto, and H. Ito, "IL-6 is required for the development of Th1 cell-mediated murine colitis," The Journal of Immunology, vol. 164, no. 9, pp. 4878-4882, 2000.

[40] E. Karatayli, R. A. Hall, S. N. Weber, S. Dooley, and F. Lammert, "Effect of alcohol on the interleukin 6-mediated inflammatory response in a new mouse model of acute-onchronic liver injury," Biochimica et Biophysica Acta (BBA)Molecular Basis of Disease, vol. 1865, no. 2, pp. 298-307, 2019.

[41] H. Yuan, Q. Ma, H. Cui et al., "How can synergism of traditional medicines benefit from network pharmacology?" Molecules, vol. 22, no. 7, p. 1135, 2017. 


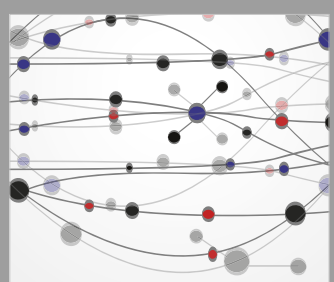

The Scientific World Journal
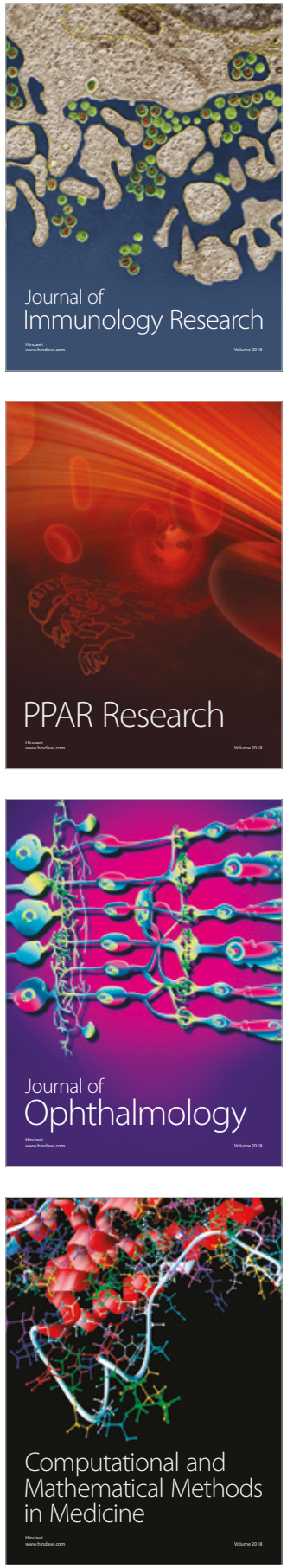

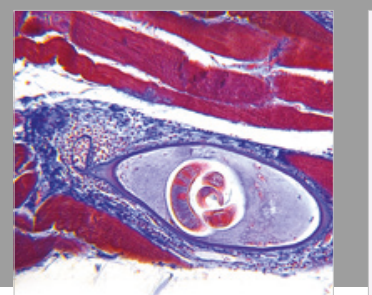

Gastroenterology Research and Practice

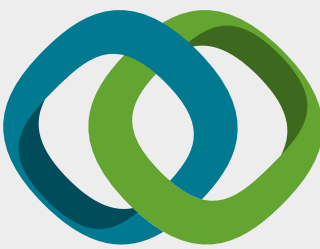

\section{Hindawi}

Submit your manuscripts at

www.hindawi.com
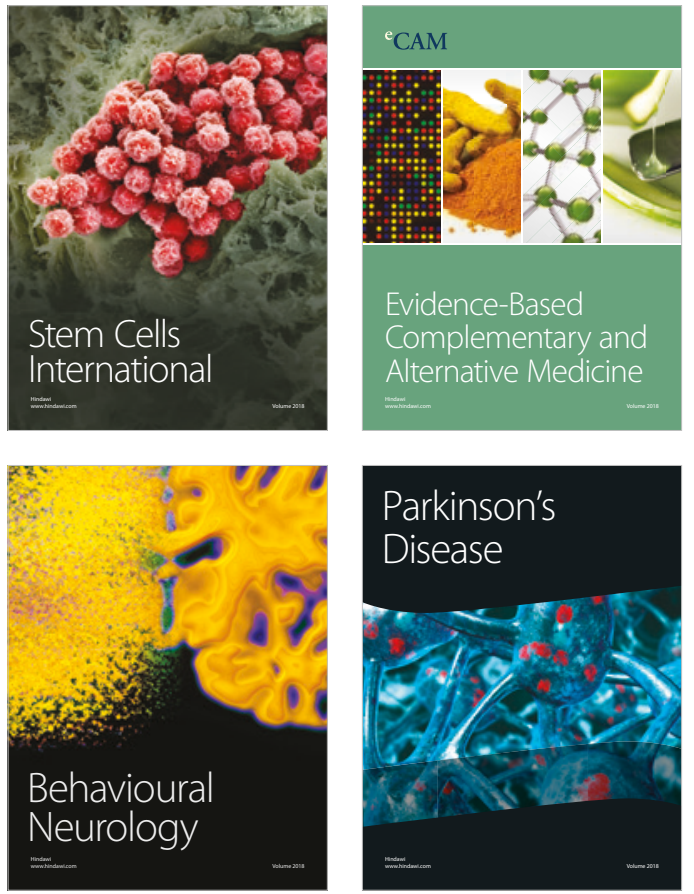

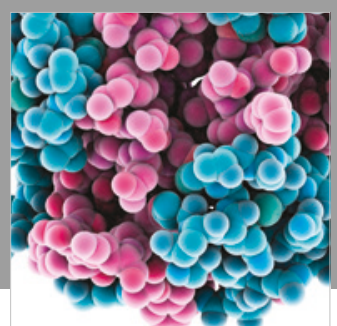

ournal of

Diabetes Research

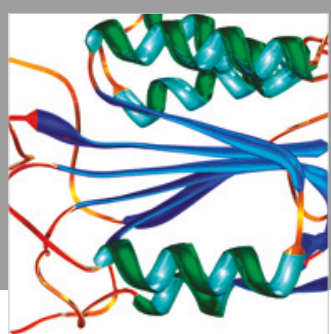

Disease Markers
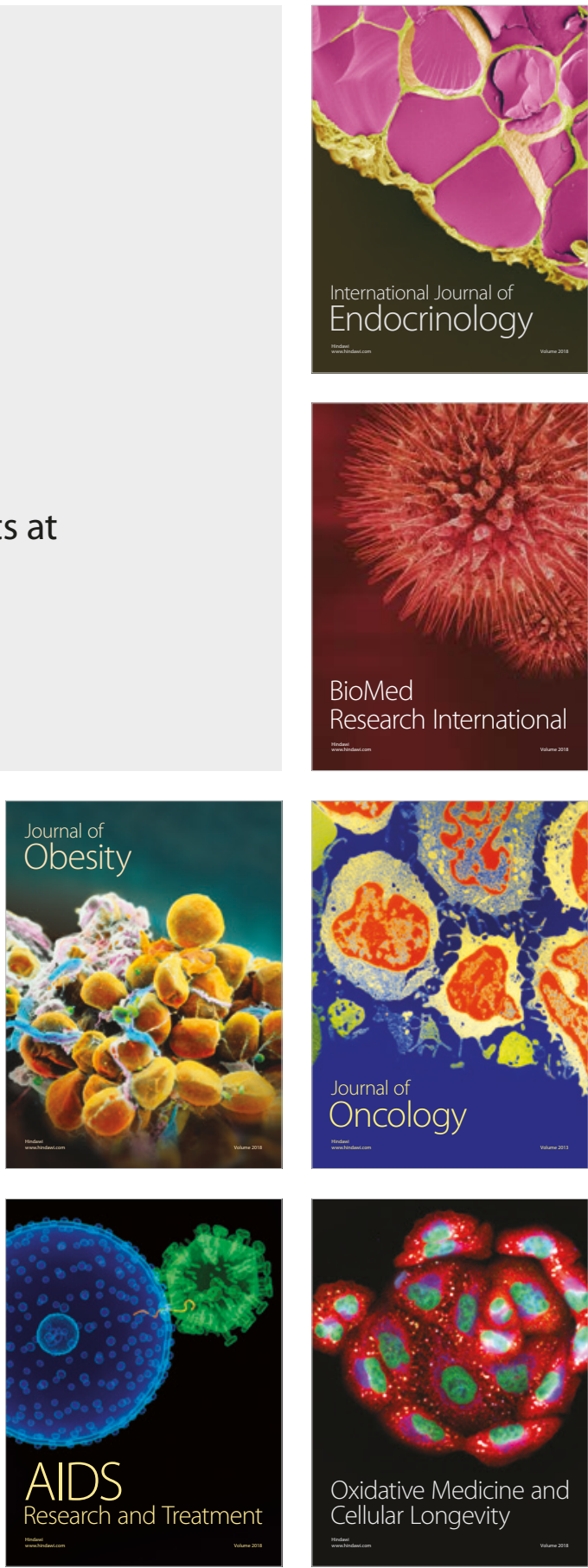